Full length article

\title{
Early identification of declining balance in higher functioning older adults, an inertial sensor based method
}

\author{
K.J. Sheehan ${ }^{\text {a,* }}$, B.R. Greene ${ }^{\text {b }}$, C. Cunningham ${ }^{a}$, L. Crosby ${ }^{a, d}$, R.A. Kenny ${ }^{a, c, d}$ \\ a Technology Research for Independent Living (TRIL), Trinity College Dublin, Dublin, Ireland \\ ${ }^{\mathrm{b}}$ Technology Research for Independent Living (TRIL), University College Dublin, Dublin, Ireland \\ c Trinity College Dublin, Department of Medical Gerontology, Dublin, Ireland \\ d St James's Hospital, Mercer's Institute for Successful Ageing, Dublin, Ireland
}

\section{A R T I C L E I N F O}

\section{Article history:}

Received 16 April 2013

Received in revised form 14 December 2013

Accepted 3 January 2014

\section{Keywords:}

Inertial sensors

Berg balance scale

Timed up and go

Balance decline

Community dwelling older adults

\begin{abstract}
A B S T R A C T
Higher functioning older adults rarely have their balance assessed clinically and as such early decline in balance is not captured. Early identification of declining balance would facilitate earlier intervention and improved management of the ageing process. This study sought to determine if (a) a once off inertial sensor measurement and (b) changes in inertial sensor measurements one year apart can identify declining balance for higher functioning older adults. One hundred and nineteen community dwelling older adults (58 males; $72.5 \pm 5.8$ years) completed a timed up and go (TUG) instrumented with inertial sensors and the Berg balance scale (BBS) at two time points, one year apart. Temporal and spatio-temporal gait parameters as well as angular velocity and turn parameters were derived from the inertial sensor data. A change in balance from baseline to follow-up was determined by sub-components of the BBS. Changes in inertial sensor parameters from baseline to follow-up demonstrated strong association with balance decline in higher functioning older adults (e.g. mean medial-lateral angular velocity odds ratio $=0.2 ; 95 \% \mathrm{CI}$ : 0.1 0.5). The area under the Receiver operating characteristic curve (AUC) ranged from 0.8 to 0.9 , a marked improvement over change in TUG time alone (AUC 0.6-0.7). Baseline inertial sensor parameters had a similar association with declining balance as age and TUG time. For higher functioning older adults, the change in inertial sensor parameters over time may reflect declining balance. These measures may be useful clinically, to monitor the balance status of older adults and facilitate earlier identification of balance deficits.
\end{abstract}

(c) 2014 Elsevier B.V. All rights reserved.

\section{Introduction}

With declining fertility and increasing life expectancy for most regions of the world, the mean global age has increased [1]. This trend is expected to continue with projected figures for 2050 of 2 billion adults over the age of 60 [2]. In order to minimise the economic and health costs of these changing population demographic early identification of decline coupled with timely medical intervention is required.

Higher functioning older adults represent those who are community dwelling, independently mobile and have few morbidities [3]. Despite evidence that it is an essential component of the functional assessment of older adults, balance is often not assessed in these older adults [4]. Declining balance is associated with limited mobility and an increased risk of falls [5]. Early detection of decline in balance could facilitate earlier intervention and potentially reduce the risk of falls. Current clinical measures of

\footnotetext{
* Corresponding author. Tel.: +353 7788555256 .

E-mail address: sheehakj@tcd.ie (K.J. Sheehan).
}

balance are restricted by ceiling scores which higher functioning older adults are likely to obtain [6]. Inertial sensor based balance assessments may represent an appropriate substitute to current assessments for higher functioning groups.

The timed up and go (TUG) is used to assess functional mobility and requires both static and dynamic balance $[7,8]$. The TUG is quick, easy to administer and has been shown to have some predictive power for falls and mobility impairment $[9,10]$. Additionally, the application of wireless inertial sensors to the lower limbs during the TUG allows measurements such as cadence, stance phase duration and angular velocity to be recorded $[11,12]$. These objective measures have been combined statistically to improve the accuracy of falls risk assessment as compared to TUG time $[11,12]$. As altered balance is associated with falls risk these inertial sensor based measures may have utility in identifying early changes in balance for higher functioning groups [4].

This study had two objectives:

(1) To determine if baseline quantitative TUG parameters are associated with a decline in balance at follow-up for higher functioning older adults. 
(2) To determine if changes in quantitative TUG parameters (measured at two time points one year apart) reflect decline in balance for higher functioning older adults.

\section{Methods}

Participants were those from the Technology Research for Independent Living (TRIL) longitudinal study on ageing (www. trilcentre.org). Those who were community dwelling, greater than 60 years of age, able to walk independently, cognitively intact and able to provide informed consent were included in the TRIL cohort. Participants attended the TRIL Clinic, St. James's Hospital Dublin, Ireland in 2010-2011 for a comprehensive baseline assessment [13]. Follow-up assessments were completed on average 12.9 months later, over a period of 22 months after the initial baseline assessment. Institutional ethical approval was granted.

In a study of several clinical measures of balance, the BBS demonstrated the lowest rates of ceiling effect amongst a group of older adults with functional limitations [5]. The BBS has been shown to be highly correlated with TUG time [12] (also referred to as manual TUG). As such it was selected as the clinical reference standard of balance for comparison with the quantitative TUG. The BBS is progressive in nature with the final components (tandem stance and single leg stance) considered the most challenging [14]. It was anticipated that a change in these more complex tasks would be seen before a change in the simpler tasks for those older adults who were higher functioning. For the current study, the more complex BBS sub-components were targeted as indices of declining balance. Decline in each BBS sub-component was defined as a negative change in component score of 2 or more $(\geq 40 \%$ reduction). Participants were categorised as 'balance declined' or 'balance not-declined' for each individual sub-component as well as overall BBS, based on how each BBS sub-component and the overall BBS changed between baseline and follow-up. For the total BBS a decline in balance was defined as a decrease in total score of at least four [15].

Participants completed the BBS and the quantitative TUG at both test sessions. The BBS was delivered and scored as per the protocol outlined by Berg et al. [16]. The TUG was conducted as per the protocol described by Greene et al. [11]. Wireless inertial sensors (SHIMMER Research, Dublin, Ireland) were used to capture kinematic data during the TUG (Fig. 1). Each sensor contained a triaxial accelerometer and a tri-axial gyroscope and sampled at $102.4 \mathrm{~Hz}$. Sensors were calibrated using a standard procedure [17] and attached to the anterior tibia of each leg. Sensor data were transmitted wirelessly via Bluetooth to a PC.

All sensor data analysis was performed using MATLAB version 7.11 (Mathworks, Natick, MA, USA). Quantitative TUG parameters were calculated from the raw sensor data, these are described in detail elsewhere [11,12]. In summary temporal, spatio-temporal, turn and tri-axial angular velocity parameters were extracted for further analyses. The sensor angular velocities were described as medio-lateral (ML), anterior-posterior (AP) and vertical (V) (Fig. 1). Quantitative TUG parameters were derived for baseline TUG sensor data and for the changes in sensor data between baseline and follow-up.

Additional measures were collected at the baseline assessment to further characterise the cohort. Cognitive status was assessed using the Mini-Mental State Examination (MMSE) [18]. Maximum grip strength was measured using a handheld dynamometer (Baseline ${ }^{\circledR}$ Hydraulic Hand Dynamometers, Nex-Gen Ergonomics Inc., Quebec, Canada). Polypharmacy was defined as the regular use of four or more prescription medications [19]. Finally, the ageadjusted Charlston co-morbidity index (AACCI) provided an indication of health status [20].

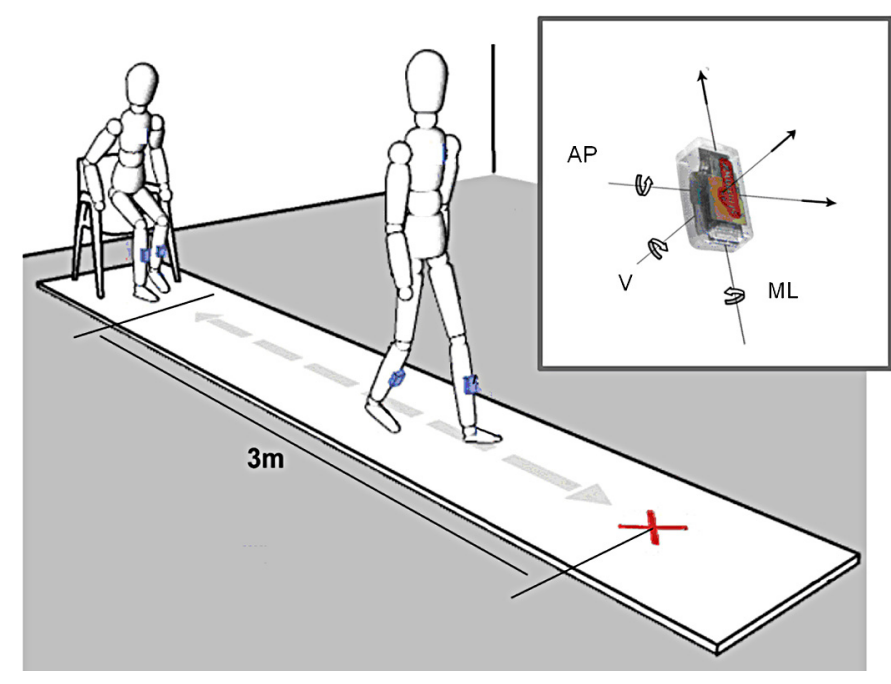

Fig. 1. Experimental setup for capture of inertial sensor data during the TUG test. Sensors were affixed to the anterior tibia of each lower limb during the TUG test.

\section{Statistical analysis}

Statistical analyses were performed using SPSS version 18.0 for Windows (SPSS Inc., Chicago, IL, USA). Baseline participant characteristics were generated for groups based on declined/ not-declined status and described in terms of sample proportions, their means and standard deviations, or median, minimum and maximum. Independent $t$-tests and Mann-Whitney rank-sum tests were performed to investigate if between groups differences $(\alpha \leq 0.05$; 95\% confidence interval (CI)) in baseline characteristics were evident. The coefficient of variation (CV) was calculated as the standard deviation of each quantitative TUG parameter divided by the mean of each quantitative TUG parameter taken across the entire TUG test.

To avoid multicollinearity the number of variables was reduced using logistic regression by block analysis. Fifty-two inertial sensor derived variables were grouped into five blocks (temporal $(n=17)$, spatio-temporal $(n=6)$, turn $(n=6)$, angular velocity $(n=14)$, angular velocity by height parameters $(n=9)$ ). The balance dichotomization of declined/not-declined for single leg stance, tandem stance and total BBS was used as the dependent variable. Working with each block, a univariate logistic regression was performed on each independent variable on each balance outcome and only those which were significant $(\alpha \leq 0.05)$ were retained in each block. Through this procedure all non-significant variables were excluded from the analyses for each final model.

Final logistic regression models were generated using the results of the sub-group analyses. Three models assessed the association of baseline quantitative TUG parameters with decline in single leg stance, tandem stance and total BBS, respectively. These models were adjusted for age as a potential confounder. An additional three models assessed whether the change in TUG parameters between baseline and follow-up assessments was associated with a decline in single leg stance, tandem stance and total BBS. Change in age over time is a constant and as such was not included in these models. An additional six univariate models assessed the association of TUG time and change in TUG time with declining balance. Odds ratios with 95\% confidence intervals for the final models were described. The area under the receiver operating characteristic (ROC) curve and its associated confidence interval was used as an indicator of each model's predictive capability. 
Table 1

Baseline characteristics for participants dichotomized into declined/non-declined groups for the sub-components and total score of the BBS.

\begin{tabular}{|c|c|c|c|c|c|c|c|}
\hline $\begin{array}{l}\text { Baseline } \\
\text { characteristic }\end{array}$ & $\begin{array}{l}\text { Total sample } \\
(n=119)\end{array}$ & $\begin{array}{l}\text { Decline single } \\
\text { leg stance } \\
(n=24)\end{array}$ & $\begin{array}{l}\text { No decline single } \\
\text { leg stance }(n=95)\end{array}$ & $\begin{array}{l}\text { Decline tandem } \\
\text { stance }(n=18)\end{array}$ & $\begin{array}{l}\text { No decline } \\
\text { tandem stance } \\
(n=101)\end{array}$ & $\begin{array}{l}\text { Decline total } \\
\text { BBS }(n=12)\end{array}$ & $\begin{array}{l}\text { No decline } \\
\text { total BBS } \\
(n=107)\end{array}$ \\
\hline \multicolumn{8}{|c|}{ Population proportions } \\
\hline Gender, M/F & $58 / 61$ & $13 / 11$ & $45 / 50$ & $9 / 9$ & $49 / 52$ & $5 / 7$ & $53 / 54$ \\
\hline Polypharmacy (\%) & 50.4 & 50.0 & 50.6 & 44.4 & 51.5 & 16.7 & 54.2 \\
\hline \multicolumn{8}{|c|}{ Mean (standard deviation) } \\
\hline Age (years) & $72.5(5.8)$ & $76.8(7.0)^{\dagger}$ & $71.4(5.0)$ & $76.8(5.7)^{\dagger}$ & $71.7(5.6)$ & $75.8(5.6)^{\dagger}$ & $72.1(5.8)$ \\
\hline Weight (kg) & $77.0(14.8)$ & $81.1(18.1)$ & $76.0(13.8)$ & $76.4(13.1)$ & $77.1(15.1)$ & $74.8(11.0)$ & $77.3(15.2)$ \\
\hline Height $(\mathrm{cm})$ & 169.3(9.0) & $167.6(9.0)$ & $169.7(9.1)$ & $169.3(6.9)$ & $169.3(9.4)$ & $166.13(6.3)$ & $169.7(9.3)$ \\
\hline TUG time $(\mathrm{s})$ & $10.4(2.4)$ & $12.3(3.0)^{\dagger}$ & $9.9(1.9)$ & $12.0(2.4)^{\dagger}$ & $10.1(2.3)$ & $12.1(3.2)^{\dagger}$ & $10.2(2.2)$ \\
\hline Grip strength (lbs) & $64.0(23.9)$ & $68.0(25.6)$ & $63.0(23.5)$ & $69.3(26.8)$ & $63.1(23.4)$ & $79.7(25.3)$ & $62.3(23.2)$ \\
\hline \multicolumn{8}{|c|}{ Median (minimum-maximum) } \\
\hline BBS & $55(45-56)$ & $53(45-55)^{*}$ & $56(48-56)$ & $53(45-55)^{*}$ & $56(45-56)$ & $53(48-55)^{*}$ & $56(45-56)$ \\
\hline MMSE & $28(18-30)$ & $28(18-30)$ & $28(19-30)$ & $28(20-30)$ & $28(18-30)$ & $28(20-30)$ & $28(18-30)$ \\
\hline AACCI & $4(2-10)$ & $4(2-8)$ & $4(2-10)$ & $4(2-8)$ & $4(2-10)$ & $5(2-7)$ & $4(2-10)$ \\
\hline
\end{tabular}

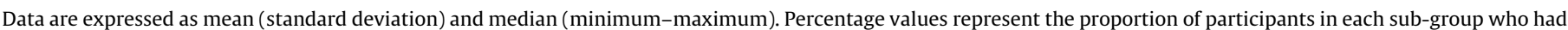
polypharmacy.

Significant between group differences were considered at the levels of ${ }^{*}$ for $\alpha \leq 0.01$ and $^{\dagger}$ for $\alpha \leq 0.05$.

Table 2

Results obtained from logistic regression models assessing the utility of baseline quantitative TUG parameters in predicting decline in balance.

\begin{tabular}{|c|c|c|c|}
\hline Model 1 & Baseline mean (SD) & Follow-up mean (SD) & Odds ratio $(95 \% \mathrm{CI})$ \\
\hline Turn mid-point time (s) & $3.8(1.0)$ & $3.9(1.0)$ & $2.8(1.5-5.5)^{*}$ \\
\hline Swing time CV (\%) & $19.0(15.0)$ & $19.6(14.9)$ & $1.0(1.0-1.1)$ \\
\hline Age & $72.5(5.8)$ & - & $1.1(1.0-1.3)^{\dagger}$ \\
\hline Model 2 & Baseline mean (SD) & Follow-up mean (SD) & Odds ratio $(95 \% \mathrm{CI})$ \\
\hline Turn mid-point time (\%) & $3.8(1.0)$ & $3.9(1.0)$ & $1.1(0.5-2.4)$ \\
\hline Angular velocity ML minimum (deg/s) & $92.5(13.2)$ & $99.2(18.8)$ & $1.1(1.0-1.1)$ \\
\hline Stance time CV (\%) & $42.7(16.7)$ & $45.3(20.6)$ & $1.0(1.0-1.1)$ \\
\hline Age & $72.5(5.8)$ & - & $1.1(1.0-1.3)^{\dagger}$ \\
\hline Model 3 & Baseline mean (SD) & Follow-up mean (SD) & Odds ratio $(95 \% \mathrm{CI})$ \\
\hline Walk time $(\mathrm{s})$ & $8.2(3.5)$ & $8.3(2.2)$ & $1.1(0.9-1.3)$ \\
\hline Angular velocity ML minimum by height ( $\operatorname{deg} \mathrm{m} / \mathrm{s}$ ) & $156.5(23.7)$ & $165.1(32.9)$ & $1.0(1.0-1.1)$ \\
\hline Age & $72.5(5.8)$ & - & $1.0(0.9-1.2)$ \\
\hline
\end{tabular}

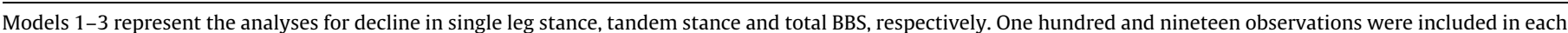
model. CV: Coefficient of variation; the ratio of the standard deviation to the mean, expressed as a percentage. SD: standard deviation. CI: confidence interval.

The odds of exhibiting a decline in balance was deemed significant at the levels of ${ }^{*} \alpha \leq 0.01$ and ${ }^{\dagger} \alpha \leq 0.05$.

\section{Results}

At baseline 447 participants were assessed. Of these 444 (99.3\%) completed the BBS and 437 (97.8\%) completed the quantitative TUG. Due to time constraints it was not possible to re-assess all participants. A convenience sample of 156 (34.9\%) participants was invited to complete a follow-up assessment. Thirteen $(8.3 \%)$ did not wish to partake, eight (5.1\%) reported being too unwell while one $(0.6 \%)$ participant reported carer burden. A further $12(7.7 \%)$ participants cancelled their appointment and did not reschedule. Three (1.9\%) participants were excluded at baseline due to poor quality sensor data. One hundred and nineteen subjects completed both assessments. There was no difference in age, cognitive status, grip strength or TUG time between those who completed follow up and those who did not $(p \geq 0.05)$. Those who were not followed up had significantly $(p \leq 0.05)$ lower BBS (median, minimum-maximum. 54, 10-56) and a trend towards a higher AACCI $(p=0.06)$.

Table 1 summarises baseline characteristics for the total sample and participants dichotomized into declined/not-declined groups. At baseline $47 \%$ of participants achieved a ceiling score on the BBS. A decline in single leg stance was seen for 24 (20.2\%) participants. Eighteen $(15.1 \%)$ declined in tandem stance and $12(10.1 \%)$ declined in total BBS. All participants who declined in total BBS declined in either single leg or tandem stance. Five participants demonstrated an improvement in total BBS. No participant improved on single leg or tandem stance. Those who presented with a decline in balance at follow-up were older, had slower baseline TUG times and lower baseline BBS $(p \leq 0.05)$. Similar baseline MMSE scores, AACCI scores, grip strengths and presence of polypharmacy ( $p \geq 0.05$ ) were seen for declined and non-declined groups.

Results from the logistic regression analyses used to determine if baseline quantitative TUG parameters were associated with a decline in balance are presented in Table 2. One baseline quantitative TUG parameter (time of turn mid-point) had a significant $(p \leq 0.001)$ relationship with a decline in balance (odds ratio 2.8 , CI: $1.5-5.5)$. Age was significantly $(p \leq 0.05)$ associated with a decline in tandem balance and single limb support (odds ratio 1.1, CI: 1.0-1.3). For a change in TUG time of one second, the odds of demonstrating a decline in balance were significant $(p \leq 0.05)$ for single leg stance (odds ratio $1.5,95 \% \mathrm{CI}: 1.2-1.9$ ), tandem stance (odds ratio $1.3,95 \% \mathrm{CI}: 1.1,1.6$ ) and total BBS (odds ratio $1.2,95 \% \mathrm{CI}: 1.1,1.3)$.

Results from the logistic regression analyses used to determine if changes in quantitative TUG parameters over time reflect change in balance are presented in Table 3. The change in five quantitative TUG parameters between baseline and follow-up (change in swing time CV, change in mean ML angular velocity, change in minimum ML angular velocity, change in mean $V$ angular velocity and change in minimum AP angular velocity by height) were significantly 
Table 3

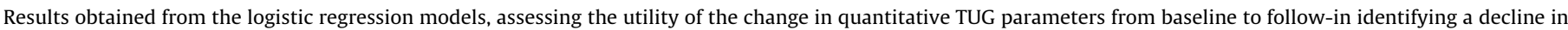
balance.

\begin{tabular}{|c|c|c|c|}
\hline Model 4 & Mean baseline (SD) & Mean follow-up (SD) & Odds ratio $(95 \% \mathrm{CI})$ \\
\hline$\Delta$ Angular velocity ML mean ( deg/s) & $20.2(3.2)$ & $25.1(4.5)$ & $0.6(0.4-0.8)^{\ddagger}$ \\
\hline$\Delta$ Angular velocity $\mathrm{V}$ mean $(\mathrm{deg} / \mathrm{s})$ & $9.3(2.1)$ & $11.5(2.6)$ & $0.7(0.5-0.9)^{\dagger}$ \\
\hline$\Delta$ Walk time $(\mathrm{s})$ & $8.2(3.5)$ & $8.3(2.2)$ & $1.3(0.9-2.0)$ \\
\hline$\Delta$ Angular velocity ML minimum (deg/s) & $92.5(13.2)$ & $99.2(18.8)$ & $0.9(0.9-1.0)^{*}$ \\
\hline$\Delta$ Swing time $\mathrm{CV}(\%)$ & $19.0(15.0)$ & $19.6(14.9)$ & $0.9(0.9-1.0)^{*}$ \\
\hline$\Delta$ Angular velocity $\mathrm{V} \mathrm{CV}(\%)$ & $151.7(18.6)$ & $138.9(16.5)$ & $1.0(0.9-1.0)$ \\
\hline$\Delta$ Angular velocity AP minimum by height $(\operatorname{deg} \mathrm{m} / \mathrm{s})$ & $308.5(86.0)$ & $311.9(81.7)$ & $1.0(1.0-1.0)^{\dagger}$ \\
\hline Model 5 & Mean baseline (SD) & Mean follow-up (SD) & Odds ratio $(95 \% \mathrm{CI})$ \\
\hline$\Delta$ Angular velocity ML mean (deg/s) & $20.2(3.2)$ & $25.1(4.5)$ & $0.2(0.1-0.5)^{\ddagger}$ \\
\hline$\Delta$ Angular velocity ML CV (\%) & $147.9(13.0)$ & $131.2(9.0)$ & $0.8(0.6-0.9)^{\ddagger}$ \\
\hline$\Delta$ Angular velocity ML minimum (deg/s) & $92.5(13.2)$ & $99.2(18.8)$ & $0.8(0.7-0.9)^{\ddagger}$ \\
\hline$\Delta$ Angular velocity ML mean during mid-swing points ( $\mathrm{deg} / \mathrm{s})$ & $117.4(16.9)$ & $124.4(18.0)$ & $1.1(1.0-1.3)^{\dagger}$ \\
\hline$\Delta$ Angular velocity AP maximum $(\mathrm{deg} / \mathrm{s})$ & $187.8(51.0)$ & $179.4(51.0)$ & $1.0(1.0-1.0)^{\dagger}$ \\
\hline Model 6 & Mean baseline (SD) & Mean follow-up (SD) & Odds ratio $(95 \% \mathrm{CI})$ \\
\hline$\Delta$ Angular velocity ML mean (deg/s) & $20.2(3.2)$ & $25.1(4.5)$ & $0.7(0.6-1.0)^{\dagger}$ \\
\hline$\Delta$ Angular velocity ML CV (\%) & $147.9(13.0)$ & $131.2(9.0)$ & $0.9(0.8-1.0)^{*}$ \\
\hline$\Delta$ Angular velocity AP CV (\%) & $155.7(19.0)$ & $137.8(16.7)$ & $1.1(1.0-1.1)^{\dagger}$ \\
\hline
\end{tabular}

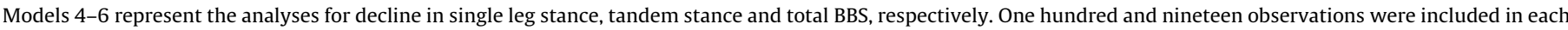

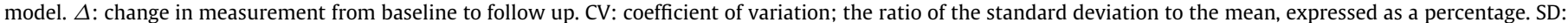
standard deviation. $\mathrm{CI}$ : confidence interval.

The odds of exhibiting a decline in balance was deemed significant at the levels of ${ }^{*} \alpha \leq 0.01,{ }^{\dagger} \alpha \leq 0.05$ and ${ }^{\ddagger} \alpha \leq 0.001$.

associated $(p \leq 0.05)$ with a decline in single leg stance. A significant association $(p \leq 0.05)$ between decline in tandem stance and the change in five quantitative TUG parameters was noted (change in maximum AP angular velocity; change in mean ML angular velocity at mid-swing points; change in CV ML angular velocity; change in minimum ML angular velocity; change in mean ML angular velocity). Between baseline and follow-up three quantitative TUG parameters changed in a manner which was significantly ( $p \leq 0.05$ ) associated with decline in total BBS (change in mean ML angular velocity; change in CV ML angular velocity; change in CV AP angular velocity). Change in TUG time between the two assessments was not significantly associated with a decline in balance (single leg stance: odds ratio $0.9,95 \% \mathrm{CI}$ : 0.9, 1.0 ; tandem stance: odds ratio $1.3,95 \% \mathrm{CI}$ : $0.9,1.7$; total $\mathrm{BBS}$ : odds ratio $1.4,95 \%$ CI: $0.9,2.1$ ).

Models 1, 2 and 3 were used to determine the association between baseline quantitative TUG parameters and decline in single leg stance, tandem stance and total BBS. From ROC analysis the predictive capabilities were 0.8 (CI: $0.8,0.9), 0.8$ (CI: 0.7, 0.9) and 0.7 (CI: $0.6,0.9$ ), respectively. While TUG time predicted a decline in balance at 0.8 (CI: 0.7,0.9) for single leg stance, 0.8 (CI: $0.6,0.9$ ) for tandem stance and 0.8 (CI: 0.5, 0.9) for total BBS.

Change in quantitative TUG parameters between baseline and follow-up were used in models 4,5 and 6 and the ROC predictive capability increased to 0.9 (CI: $0.8,1.0$ ) (single leg stance), 0.9 (CI: $0.9,1.0)$ (tandem stance) and 0.8 (CI: 0.6, 0.9) (total BBS) (Fig. 2). The predictive capability of change in TUG time between the two assessments for a decline in balance was 0.6 (CI: $0.4,0.7)$ for single leg stance, and 0.7 (CI: $0.6,0.8)$ for tandem stance and total BBS, respectively.

\section{Discussion}

This is the first study to assess the utility of inertial sensor parameters recorded during the TUG test for the early identification of declining balance for higher functioning older adults. Baseline quantitative TUG parameters did not represent an improvement over age or TUG time in identifying those older adults who exhibited a decline in balance. The changes from baseline to follow-up seen for quantitative TUG parameters were strongly associated with a decline in balance. This was not the case for a change in TUG time alone. This suggests that the changes in quantitative TUG parameters over time reflect an early decline in balance.

Baseline characteristics indicated the group as a whole was high functioning with high baseline BBS, grip strength and MMSE scores, low TUG times and AACCI scores. Participants who demonstrated a decline in balance were at a similar cognitive level, with a similar degree of co-morbidity, medication use and muscle strength as their peers who did not decline in balance $(p>0.05)$. Lower baseline BBS scores were seen for declined groups $(p \leq 0.05)$. This was to be anticipated as a change in BBS sub-component scores would be more likely for those who did not achieve a ceiling score at baseline [5]. The quantitative parameters were capable of reflecting this change and provide the clinician with additional objective information relating to this change. Participants who declined also had longer baseline TUG times and were older. This reflects the high correlation between the BBS, TUG time and age $[7,21]$.

An increase in the time taken to complete the first half of the TUG at baseline (Turn mid-point time) was associated with a decline in balance $(p<0.001)$. However the time taken to complete the second half of the TUG was not associated with a decline in balance. Previous research indicated that the time taken to complete the second half on the TUG was associated with cognitive decline in community dwelling older adults [21]. This highlights the potential of a sensor based TUG test to discriminate early balance from early cognitive deficits. From the ROC analyses baseline quantitative TUG parameters showed similar predictive capabilities as TUG time. When looking at the models closer the odds of exhibiting a decline in balance per unit change of quantitative TUG parameters was greater than that seen for age or TUG time. For example, for a one second increase in the time of the turn mid-point, the likelihood a decline in balance was 2.8 times for single leg stance, while for each advancing year of age an individual was 1.1 times more likely to decline in single leg stance. This suggests that while the overall associations were similar, the baseline quantitative TUG parameters may be more sensitive.

The change in quantitative TUG parameters over time was strongly associated with declining balance. An increase in ML angular velocity at mid swing points was associated with a decline 

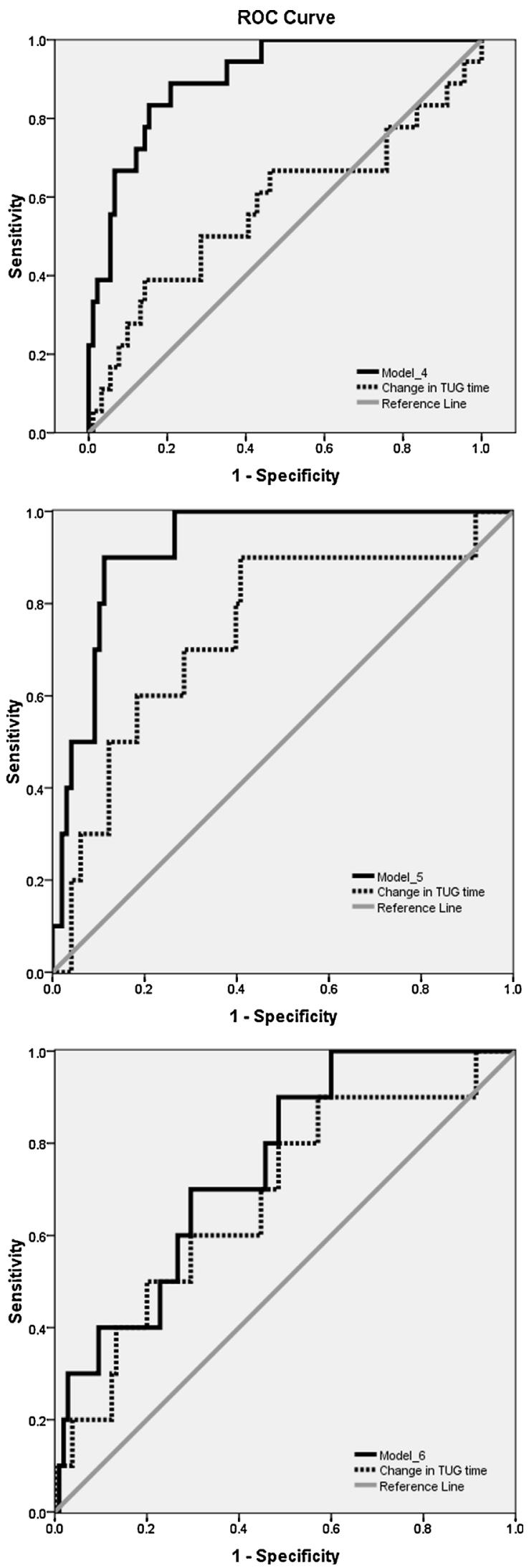

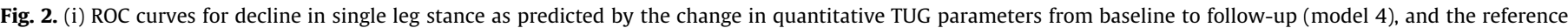

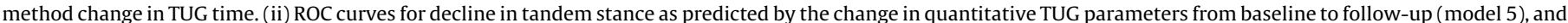

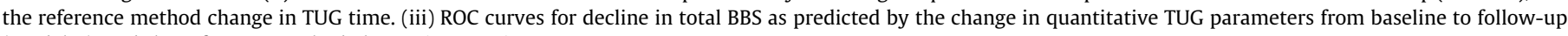
(model 6), and the reference method change in TUG time. 
in balance. This association may be due to an impaired ability to decelerate the swinging limb as it enters terminal swing and prepares for load acceptance. In contrast an increase in other angular velocity measures was associated with a reduced likelihood of declining balance. This finding was anticipated as it is reported that a shorter TUG time is associated with more positive health outcomes $[9,10]$.

The current study also investigated the relationship between variance and the change in variance over time for gait parameters, and change in balance over time. The coefficient of variation associated with angular velocity parameters was seen to be $>100 \%$ for the $X, Y$ and $Z$ sensor axes at baseline and follow-up assessments. This indicates that these parameters are highly variable between one stride and the next. An increase in the variance of stride time is associated with poor balance and an increased falls risk for older adults [22]. For the current study a change in ML and AP angular velocity between baseline and follow up assessments was associated with a decline in balance. The results suggest that stride-to stride adjustments in medio-lateral angular velocity facilitate balance function whereas an increase in anterior-posterior variance indicates an impairment in balance function.

For the present study a change in mean ML angular velocity, minimum ML angular velocity, ML angular velocity at mid swing points, and mean $\mathrm{V}$ angular velocity from baseline to follow-up assessment was indicative of a decline in balance. All four of the these parameters are associated with minimum ground clearance (MGC) and may discriminate fallers from non fallers [23,24].

With a high proportion of the sample achieving ceiling scores on the BBS at baseline, the ability to detect a change in balance status was restricted. The results presented here are encouraging. Despite relatively few participants exhibiting a change in balance at follow-up, several quantitative TUG parameters were associated with declining balance. Sample size may also explain why some parameters demonstrated larger odds ratios but were nonsignificant. A longer follow-up period would increase the number of participants who demonstrate declining balance and further highlight the potential benefits of inertial sensor technology. Finally, using changes on a five point sub-component scale as a proxy for declining balance is not without limitations. The subcomponents are also subject to ceiling effects and have scores which are under utilised [25]. As such a conservative dichotomized approach was adopted here with a reduction of at least $40 \%$ in component score considered a true change. This may have led to an underestimation of the benefits of this method. Future study may further assess the hypotheses presented here using a force plate to record centre of pressure as a measure of balance, which would negate the ceiling effects issue.

Current clinical measures of balance are restricted to those with moderate or severe functional limitations as a result of ceiling effects. For higher functioning older adults TUG inertial sensor parameters represent an objective alternative to current clinical measures of balance. The present method is a time effective means of monitoring changes in balance over time. The regular measurement of balance in a primary care setting could facilitate the early identification of declining functional status, potentially enabling timely intervention.

\section{Acknowledgements}

This research was completed as part of a wider programme of research within the TRIL Centre, (Technology Research for Independent Living). The TRIL Centre is a multi-disciplinary research centre, bringing together researchers from TCD, UCD,
NUIG and Intel http://www.trilcentre.org. This research was funded by Intel, GE Healthcare and IDA Ireland. We also acknowledge the help and support of the staff of the TRIL Clinic, St James's Hospital, Dublin and the participants involved in this study.

\section{Conflict of interest statement}

Author BRG is a director of a company with a license to commercialise the technology reported in this study.

\section{References}

[1] Mathers CD, Loncar D. Projections of global mortality and burden of disease from 2002 to 2030. PLoS Med 2006; 3:442.

[2] Harper S. Addressing the implications of global ageing. J Pop Res 2006;23: 205-23.

[3] Lowry KA, Vallejo AN, Studenski SA. Successful aging as a continuum of functional independence: lessons from physical disability models of aging. Aging Dis 2012;3:5-15.

[4] Howe TE, Rochester L, Jackson A, Banks PM, Blair VA. Exercise for improving balance in older people. Cochrane Database Syst Rev 2009;4:CD004963.

[5] Muir SW, Berg K, Chesworth B, Speechley M. Quantifying the magnitude of risk for balance impairment on falls in community-dwelling older adults: a systematic review and meta analysis. J Clin Epidemiol 2010;63:389-406.

[6] Pardasaney KP, Latham NK, Jette AM, Wagenaar RC, Pengsheng N, Slavin MD, Bean JF. Sensitivity to change and responsiveness of four balance measures for community-dwelling older adults. Phys Ther 2012;92:388-97.

[7] Podsiadlo D, Richardson S. The timed 'up \& go': a test of basic functional mobility for frail elderly persons. JAGS 1991;31:142-8.

[8] Mathias S, Nayak U, Saacs B. Balance in elderly patients: the get-up and go test. Arch Phys Med Rehabil 1986;67:387-9.

[9] Bischoff HA, Stahelin HB, Monsch AU, Iversen MD, Weyh A, von Dechend M, Akos R, Conzelmann M, Dick W, Theiler R. Identifying a cut-off point for normal mobility: a comparison of the timed 'up and go' test in community-dwelling and institutionalised elderly women. Age Ageing 2003;32:315-20.

[10] Shumway-Cook A, Brauer S, Woollacott M. Predicting the probability for falls in community-dwelling older adults using the timed up \& go test. Phys Ther 2000;80:896-903.

[11] Greene BR, O’Donovan A, Romero-Ortuno R, Cogan L, Scanaill CN, Kenny RA Quantitative falls risk assessment using the timed up and go test. IEEE Trans Biomed Eng 2010;57:2918-26.

[12] Greene BR, Doheny EP, Walsh C, Cunningham C, Crosby L, Kenny RA. Evaluation of falls risk in community-dwelling older adults using body-worn sensors. Gerontology 2012;58:472-80.

[13] Romero-Ortuno R, Cogan L, Foran T, Kenny RA, Fan CW. Continuous noninvasive orthostatic blood pressure measurements and their relationship with orthostatic intolerance, falls and frailty in older people. JAGS 2011;59:655-65.

[14] Berg KO, Wood-Dauphinee SL, Williams JI, Gayton D. Measuring balance in the elderly: preliminary development of an instrument. Phys Can 1989;41: 304-10.

[15] Donoghue D, Stokes EK. How much change is true change? The minimum detectable change of the Berg balance scale in elderly people. J Rehabil Med 2009;41:343-6.

[16] Berg KO, Wood-Dauphinee SL, Williams JI, Maki B. Measuring balance in the elderly: validation of an instrument. Can J Pub Health 1992;83:S7-11

[17] Ferraris F, Grimaldi U, Parvis M. Procedure for effortless in-field calibration of three-axis rate gyros and accelerometers. Sens Mater 1995;7:311-30.

[18] Cockrell JR, Folstein MF. Mini-mental state examination; principles and practice of geriatric psychiatry. New York, NY: Wiley; 2002. p. 140-1.

[19] Denneboom W, Dautzenberg MG, Grol R, De Smet PA. Analysis of Polypharmacy in older patients in primary care using a multidisciplinary expert panel. Br J Gen Pract 2006;56:504-10.

[20] Charlson M, Szatrowski TP, Peterson J, Gold J. Validation of a combined comorbidity index. J Clin Epidemiol 1994;47:1245-51.

[21] Greene BR, Kenny RA. Assessment of cognitive decline through quantitative analysis of the timed up and go test. IEEE Trans Biomed Eng 2012;59:988-95

[22] Hausdorff JM, Rios DA, Edelberg HK. Gait variability and fall risk in community-living older adults: a 1-year prospective study. Arch Phys Med Rehabil 2001;82:1050-6.

[23] Greene BR, McGrath D, Foran TG, Doheny EP, Caulfield B. Body-worn sensor based surrogates of minimum ground clearance in elderly fallers and controls. In: International Conference of the IEEE EMBS; 2011.

[24] McGrath D, Greene BR, Walsh C, Caulfield B. Estimation of minimum ground clearance (MGC) using body-worn inertial sensors. J Biomech 2011;44:1083-8

[25] Kornetti DL, Fritz SL, Chiu Y, Light KE, Veloza CA. Rating scale analysis of the Berg balance scale. Arch Phys Med Rehabil 2004;85:1128-35. 